\title{
Performance Analysis of DWDM System with Optical Amplifiers in Cascade Considering the Effect of Crosstalk
}

\author{
Abu Jahid*, Sanwar Hossain, Raziqul Islam \\ Dept. of Electrical and Electronic Engineering, Bangladesh University of Business and Technology (BUBT), Dhaka, Bangladesh \\ Email address: \\ setujahid@gmail.com (A. Jahid), sanwar_05eee@yahoo.com (S. Hossain), raziqul@yahoo.com (R. Islam) \\ To cite this article: \\ Abu Jahid, Sanwar Hossain, Raziqul Islam. Performance Analysis of DWDM System with Optical Amplifiers in Cascade Considering the \\ Effect of Crosstalk. Journal of Electrical and Electronic Engineering. Vol. 3, No. 5, 2015, pp. 110-116. doi: 10.11648/j.jeee.20150305.12
}

\begin{abstract}
In this paper, an analytical approach is presented to evaluate the performance of DWDM system with intensity modulation direct detection (IM/DD) due to the effects of amplified spontaneous emission (ASE) noise of optical amplifier, optical receiver noises and crosstalk. And a system has been proposed with an optimum number of amplifiers with higher gain and an improved optical receiver has been proposed. We have investigated the effects of optical amplifiers and optical receiver in the presence of crosstalk on the overall performance; in particular, Signal-to-Noise Ratio (SNR) and Bit-Error-Rate (BER) performance of a DWDM system. The system performances are evaluated for varying different amplifier gains, number of optical amplifiers, hop length, number of hops, receiver bandwidth and receiver gain considering the relationship between crosstalk and the number of wavelengths channel spacing. It is found that, the performance is highly degraded due to crosstalk and noises. As a result the system suffers significant power penalty at a given BER.
\end{abstract}

Keywords: ASE, IM/DD, BER, Crosstalk, Power Penalty

\section{Introduction}

Dense Wavelength Division Multiplexing (DWDM) is a rapidly maturing transmission technique to satisfy substantial increase of telecommunication and optical network capacity. In recent year, tremendous rate of traffic growth due to the demands for multimedia services urges the development of wavelength routing technologies in place of the point-to-point multi-wavelength transmission. In a WDM system, the channel capacity can be increased by assigning each input signals to a particular wavelength of a light. An appropriate channel filtering and proper switching scheme reduce delay time of WDM signals at the receiver output and the resultant capacity is the aggregate of carrying capacity of each signals. By using WDM technology in optical networks, $50 \mathrm{THz}$ link capacity can be achieved. [1] In order to access the huge bandwidth capacity and to utilize it effectively, DWDM is one of the promising techniques over Coarse WDM (CWDM) technology. In DWDM, the wavelength spacing $(0.8 \mathrm{~nm})$ is much closer than CWDM $(20 \mathrm{~nm})$ and therefore DWDM has greater capacity.

Now a days DWDM system supports 160 wavelengths transmitting at a speed of $10 \mathrm{Gbps}$. So the total system capacity is $1.6 \mathrm{Tbps}$ whereas CWDM provides only 50Gbps. [2] One of the significant key features of DWDM is the ability to amplify all the incoming signals with different wavelengths at once without any optical to electrical conversion. It implies that DWDM combines multiple optical signals so that they can be amplified as a group and transmitted over a single fiber to increase the capacity. Another important feature is that DWDM link can carry each signal in a different rate and in a different format. [3]

In order to increase the data transmission efficiency of DWDM systems optical amplifiers plays a vital role for optical communication system. In order to compensate fiber losses and to achieve successful data transmission multiple optical amplifiers are installed along the fiber link. An optical amplifier has the ability to amplify, regenerate and to synchronize data of optical signals up to destination. The key advantages of optical amplifiers is to provide large bandwidth and to exhibit low noise, low insertion loss at third transmission window (1550 $\mathrm{nm}$ range). [4] Erbium-Doped Fiber Amplifier (EDFA) operates in C-band region in the range of $1540 \mathrm{~nm}$ to $1565 \mathrm{~nm}$ wavelength. EDFA enables to amplify all wavelengths to overcome loss over long spans of fiber, high passive losses and to minimize dispersion effects. [5, 6] EDFA can be employed in where the application requires high bit rates, low noise and high output power. Due to wide amplification spectrum band EDFA is widely used. [7, 8] 
Amplified Spontaneous Emission (ASE) noise accumulates to the signal during its amplifications, which deteriorates the signal-to-noise ratio (SNR) and hence increase the bit error rate (BER). ASE beat noise is the dominant over the thermal noise and other receiver noises too. [9] Unequal gain spectrum of optical amplifiers is the major limitation during amplification. This mean that all wavelengths are not equally amplified which limits the performance. [10]

For a successful and efficient data reception the transmitted power should be large enough. The receiver sensitivity is the minimum amount of power requirement from the transmitter and is used to separate each single bit of 1's and 0's from the original input signal at receiving end. $[11,12]$ The acceptable BER is $10^{-9}$ in optical communications. Despite the attenuation along the optical fiber the transmitted power should be high enough to maintain the target BER. The large amount of transmitted power generates channel impairments and nonlinearities which are not expected. As a result, a high input power does not ensure that the reception would be successful. Thus the input power value should meet the minimum power requirement at the receiver. [13, 14] The receiver must be operated in the dynamic range for the incoming optical signals. However, the transmission length increases with the increase in the bit rate and the parameters have the capability of absenting in the network. [15]

Crosstalk is the power leakage from other channels at the same or different nominal wavelength on the signal channel. It occurs when signals from one channel to arrive in another channel and can be considered as interference. Crosstalk occurs due to the optical filters, wavelength multiplexers and demultiplexers, switches, optical amplifiers, and the fiber itself in a WDM link. Crosstalk has serious effect on the system performance because it reduces SNR and hence BER is increased. Crosstalk is one of the major impairments in optical networks. Theoretical result shows that for a given value of input power crosstalk increases with the increase of number of wavelength. Thus it limits the allowable number of channels per fiber. Crosstalk can be classified into two categories as intra-band or homodyne and inter-band or heterodyne crosstalk.

\section{[16-19]}

In this paper, BER performance of DWDM system with optical amplifiers in cascade incorporated with optical crosstalk has been analyzed. Simulations are performed to evaluate the impact of crosstalk and noises on the SNR and BER. The paper has been organized as following, section I describes the introduction, section covers the system model, mathematical analysis is presented in section III, section IV discusses the results and discussion and finally conclusion has been presented in section $\mathrm{V}$.

\section{System Model}

A typical block diagram of Dense Wavelength Division Multiplexing (DWDM) system with various components is shown in Fig. 1. It consists of $M$ number of transmitters, an optical waveguide which would be the medium of transmission with cascading a numbers of optical amplifiers and $\mathrm{N}$ numbers of receiver to reproduce that transmitted signal. Most practical optical communication links use laser diodes as transmitters and $\mathrm{P}-\mathrm{i}-\mathrm{N}$ or Avalanche Photodiodes (APD) as receivers. A transmitter converts an electrical signal into an optical signal whereas an optical receiver does the reverse operation. These devices modulate and detect solely the intensity of carriers not their phase which implies that all transmitted signal intensities are non-negative. The optical signals from different WDM channels are multiplexed by a WDM multiplexer. Before sending the resultant WDM signals to the transmission fiber link, a power amplifier is used to increase its output power. A number of line amplifiers are installed in cascade along the fiber link to compensate fiber losses. The definite interval can be defined as the distance between two successive line amplifiers and this length is typically $60-120 \mathrm{~km}$. The individual signal can be extracted from the multiplexed channel by using appropriate channel tuning. To provide high receiver sensitivity and high gain, optical amplifiers are used in front of demultiplexer. Therefore, a faithful and successful reception from the demultiplexed signals can be obtained.

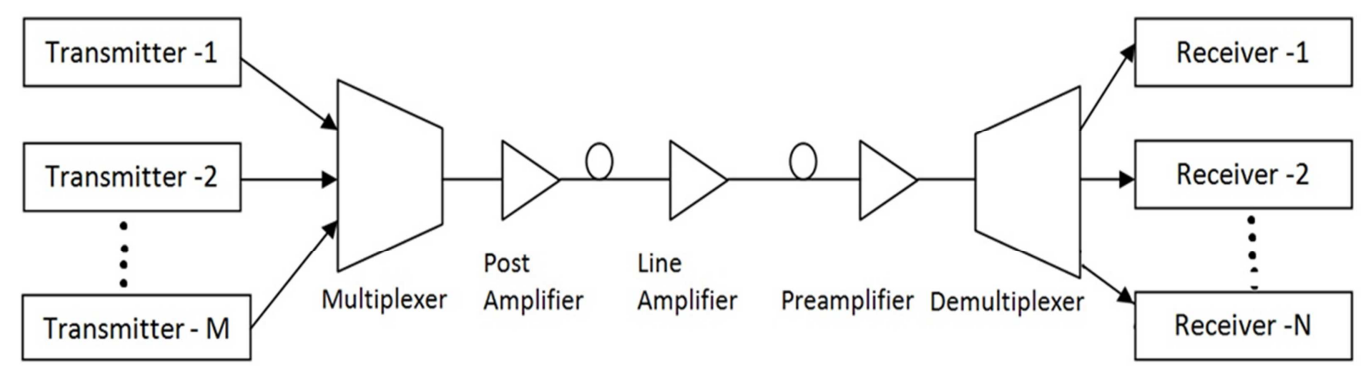

Figure 1. DWDM system with optical amplifiers in cascade.

\section{Mathematical Model}

Crosstalk is a major limiting factor to the implementation of cascaded optical amplifier in DWDM systems. It is further noticed that other receiver noises like shot noise, dark current noise, thermal noise and Amplified Spontaneous Emission
(ASE) noise are also accumulated during the optical transmission through the fiber link.

The discrete nature of electrons in photodiode generates shot noise. It can be expressed as: [20]

$$
\mathrm{i}_{\mathrm{s}}=\sqrt{2 e(I p) B}
$$


Where $\mathrm{i}_{\mathrm{S}}$ is the shot noise current, Ip is the photocurrent and $\mathrm{B}$ is the photodiode bandwidth.

Spontaneous fluctuations due to thermal interaction between free electrons and vibrating ions inside an optical waveguide create thermal noise and it can be defined as-

$$
\mathrm{i}_{\mathrm{t}}=\sqrt{4 k T B / R_{L}}
$$

Where $i_{t}$ is thermal noise current, $k$ is the Boltzmann constant $\left(1.38 * 10^{-23} \mathrm{~J} / \mathrm{K}\right), \mathrm{R}_{\mathrm{L}}$ is the load resistance and $\mathrm{T}(\mathrm{K})$ is the absolute temperature. The effect of thermal noise can be minimized by increasing the value of load resistance and the value of receiver bandwidth should be limited.

A very small amount of reverse leakage current flows in the receiver when there is no optical power is incident on the receiver is named as dark current noise. It is given by-

$$
\mathrm{i}_{\mathrm{d}}=\sqrt{2 e\left(I_{D}\right) B}
$$

The spontaneous recombination of electrons and holes in the amplifier medium is mainly responsible for ASE noise. This recombination generates a broad spectral background of photons that gets amplified along with the optical signal. The Power Spectral Density (PSD) of the ASE noise is- [14]

$$
\mathrm{N}_{\mathrm{sp}}(\mathrm{f})=\mathrm{n}_{\mathrm{sp}}(\mathrm{G}-1) \mathrm{hf}=\mathrm{Khf}
$$

Where,

$\mathrm{n}_{\mathrm{sp}}=$ Spontaneous emission factor, $\mathrm{G}=$ Amplifier gain, $\mathrm{h}=$ Plank's constant, $\mathrm{f}=$ Frequency of radiation.

In order to compensate fiber losses, optical amplifiers in cascaded form are needed to be used along the fiber link despite the ASE noise is generated. The gain of optical amplifier should be such that it can balance the losses along the optical fibers. The span length solely depends on number of optical amplifiers. ASE noise limits the hop length as well as the number of optical amplifiers. In order to receive a strong signal at the output, optimum numbers of amplifiers are used with larger gain. Thus it makes the receiver sensitivity higher and minimizes the cost.

If the amplifiers gain $(\mathrm{G})$ is adjusted to compensate for the total losses, then

$$
G(d B)=P_{L}(d B)=\left(\alpha f c+\alpha j_{j} L(d B)\right.
$$

Where, $\alpha_{\mathrm{fc}}=$ Fiber cable loss $(\mathrm{dB} / \mathrm{km}), \alpha_{\mathrm{j}}=$ Joint $\operatorname{loss}(\mathrm{dB} / \mathrm{km})$, $\mathrm{L}=$ Hop length (in $\mathrm{km}$ )

Then total amplified spontaneous emission noise at the input of the receiver is,

$$
\mathrm{P}_{\mathrm{ASE}}=\mathrm{NKhfB}
$$

Where $\mathrm{N}$ is the total number of amplifiers and $\mathrm{N}=\mathrm{L}_{\mathrm{t}} / \mathrm{L}$, $\left[\mathrm{L}_{\mathrm{t}}=\right.$ Total transmission distance in $\left.\mathrm{km}\right]$

With the addition of crosstalk, the total noise current is now

$$
\text { In }=\left(i_{\mathrm{s}}^{2}+\mathrm{i}_{\mathrm{d}}^{2}+\mathrm{i}_{\mathrm{t}}^{2}+\mathrm{P}_{\mathrm{c}}\right) 1 / 2
$$

Where $\mathrm{P}_{\mathrm{c}}$ is the crosstalk power.
The signal to noise ratio is defined as the ratio of signal power to the noise power. It is given by the following equation-

$$
\mathrm{SNR}=\frac{I_{p}^{2}}{I_{n}^{2}}
$$

The SNR for the $p-i-n$ photodiode receiver may be obtained by summing the noise contributions from Equation 1, Equation 2, Equation 3 and Equation 5. It is given by from Equation 7:

$$
\frac{S}{N}=\frac{I_{P}^{2}}{2 e B\left(I_{P}+I_{D}\right)+\frac{4 k T B F_{n}}{R_{L}}+P_{A S E}+P_{C}}
$$

Where $F_{n}$ represents the noise figure.

It is observed from equation (8), without internal avalanche gain thermal noise is dominates over the shot noise and dark current noise.

In the Avalanche Photodiode (APD) the signal current is increased by a factor $\mathrm{M}$ and hence the overall SNR is increased as thermal noise remains unaffected. But the multiplication factor (M) increases the dark current noise and quantum noise which may be a limiting factor. The overall signal-to-noise ratio for APD receiver can be expressed as[20]

$$
\frac{S}{N}=\frac{I_{P}^{2} M^{2}}{2 e B\left(I_{P}+I_{D}\right) M^{2+x}+\frac{4 k T B F n}{R_{L}}+P_{A S E}+P_{C}}
$$

Where the factor $x$ ranges between 0 and 1.0 and depending on photodiode material.

It is apparent from Eq. (9) that the first term in the denominator increases with increasing. For lower $M$ the combined thermal and amplifier noise term dominates and the total noise power is virtually unaffected when the signal level is increased, giving an improved SNR. However, when $M$ is large, the thermal and amplifier noise terms become insignificant and the SNR decreases with increasing $M$ at the rate of $M^{x}$. Therefore, an optimum value of the multiplication factor $M$ should be used.

Therefore Bit Error Rate (BER) in terms of SNR can be written as:

$$
\mathrm{BER}=0.5 \operatorname{erfc}\left[\frac{\sqrt{\frac{S}{N}}}{2 \sqrt{2}}\right]
$$

The BER depends on noise as well as other impairments on the system. It should be noted that a high SNR means a higher power signal compared to the noise power and a lower the bit error rate means less error probability.

\section{Results and Discussions}

From the mathematical model discussed in section III, the performance of DWDM system in the presence of crosstalk has been analyzed using graphical representation. The system performance has been demonstrated varying different 
parameters like number of amplifiers, amplifier gains, number of hops and hop length. We also quantified the results in terms of optical receiver type and system bandwidth. We used MATLAB tool for this simulation. Theoretical analysis has been carried out for the system for a given set of parameters and finally the system performance has been plotted for different parameters. The set of parameters are as follows for which we have used some fixed values in our experiment:

Wavelength $(\lambda)=1550 \mathrm{~nm}$, Spontaneous Emission factor $\left(\mathrm{n}_{\mathrm{SP}}\right)=1.85$, Load resistance $\left(\mathrm{R}_{\mathrm{L}}\right)=4 \mathrm{k} \Omega$, Dark current $\left(\mathrm{i}_{\mathrm{D}}\right)=$ $3 \mathrm{nA}$, Crosstalk power density $=10^{-14}$, Noise figure $\left(\mathrm{F}_{\mathrm{n}}\right)=3 \mathrm{~dB}$.

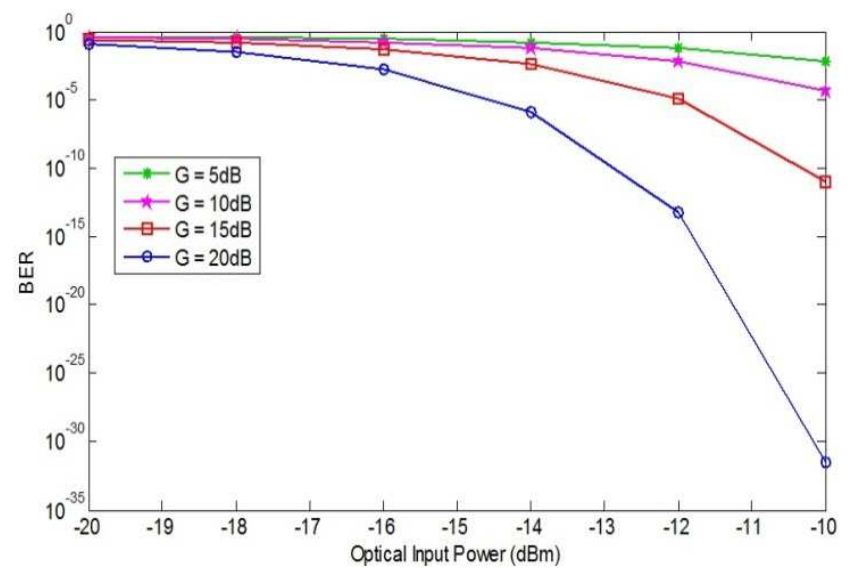

Figure 2. BER vs optical input power varying amplifier gain $(G=5 \mathrm{~dB}, 10 \mathrm{~dB}$, $15 d B, 20 d B)$ for a fixed number of amplifier $(N=10)$.

Figure 2 demonstrates the variation of BER with an optical power for different amplifier gain of $5 \mathrm{~dB}, 10 \mathrm{~dB}, 15 \mathrm{~dB}$ and $20 \mathrm{~dB}$. The BER is decreases with the increase of optical input power. The BER is highest for the amplifier gain of $5 \mathrm{~dB}$. So the BER is decreases with the increase of amplifier gain.

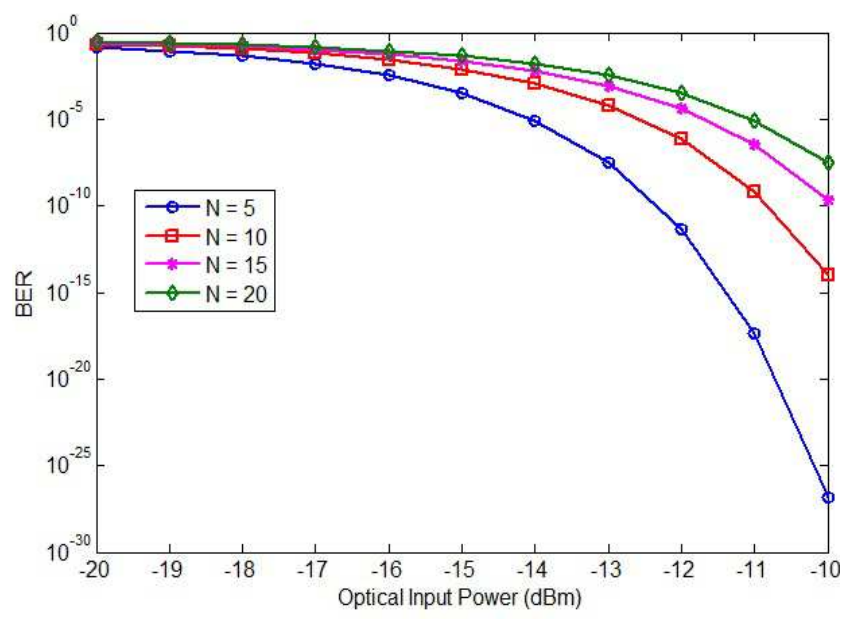

Figure 3. BER vs optical input power varying different number of amplifiers $(N=5,10,15,20)$ for a fixed gain of $G=10 \mathrm{~dB}$.

In Figure 3, BER is plotted against optical input power varying different number of amplifiers for a fixed gain of $10 \mathrm{~dB}$. It shows that, the BER is lowest when number of amplifiers is 5. Thus the highest number of amplifiers produces highest BER.

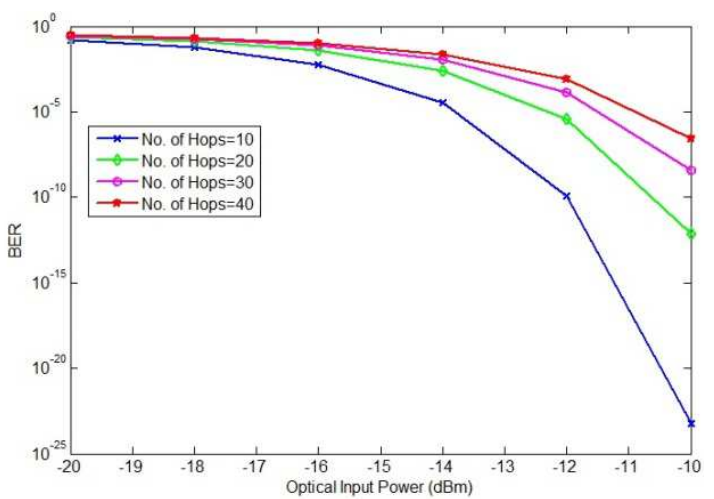

Figure 4. BER vs optical input power for different number of hops $(N h=10$, $20,30,40)$ with a fixed gain of $G=10 \mathrm{~dB}$ and bandwidth, $B=5 \mathrm{GHz}$.

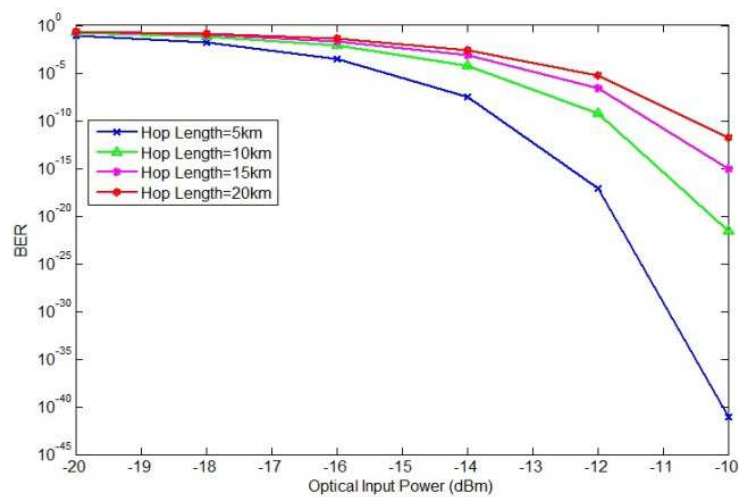

Figure 5. BER vs optical input power varying hop length of $5 \mathrm{~km}, 10 \mathrm{~km}, 15 \mathrm{~km}, 20 \mathrm{~km}$ for a fixed gain of $G=10 \mathrm{~dB}$ and bandwidth, $B=5 \mathrm{GHZ}$.

In Figure 4, we present a graphical representation of BER vs received optical power varying different number of hops is $10,20,30$, and 40 . It shows, the BER is highest when the number of hops is 40 . So it is clear that with the increase of number of hops the BER is also increases. In Figure 5, BER is plotted against optical received power for different hop length. The plot depicts that the BER is lowest for the hop length of $5 \mathrm{~km}$. So as the hop length is increases the BER is also increase. Comparing Figure 4 and Figure 5, for a fixed value of gain $10 \mathrm{~dB}$ and a bandwidth of $5 \mathrm{GHz}$, higher number of hops produces higher BER than high values of hop length. So it can be concluded that higher hop length is more preferable than higher number of hops.

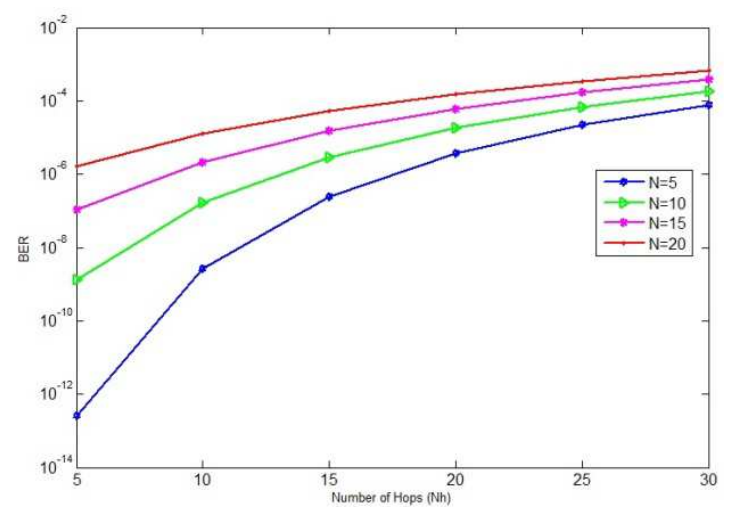

Figure 6. BER vs number of hops varying number of amplifiers $(N=5,10,15$, 20) for a fixed gain of $G=10 \mathrm{~dB}$ and bandwidth $B=5 \mathrm{GHz}$. 
In Figure 6, BER vs number of hops has been plotted varying number of amplifiers. The BER increases with the increase of number of hops and also with the increase of number of optical amplifiers. Thus with the increase of the value of both parameters, BER performance is very inferior.

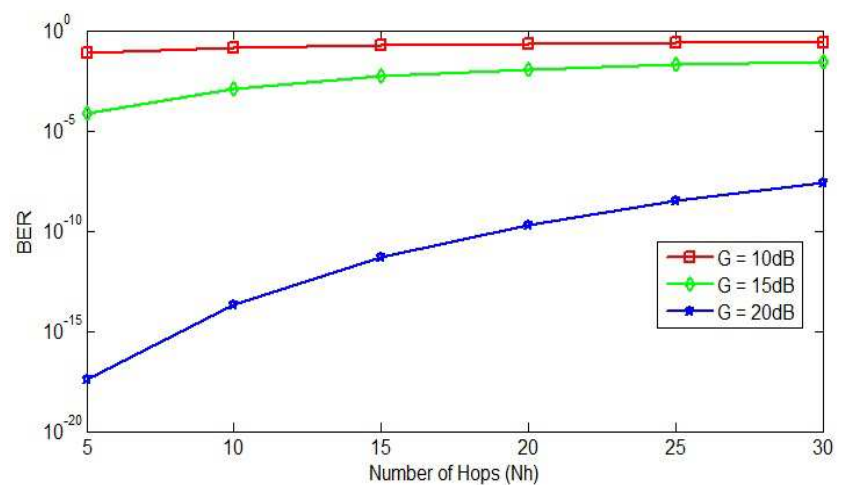

Figure 7. BER vs number of hops varying amplifier gain $(G=10 \mathrm{~dB}, 15 \mathrm{~dB}$, $20 \mathrm{~dB}$ ) for a fixed number of amplifiers of $N=10$, optical received power $=$ $5 \mathrm{dBm}$, bandwidth, $\mathrm{B}=5 \mathrm{GHz}$.

In figure 7 , BER vs number of hops varying amplifier gain for a fixed number of amplifiers of $\mathrm{N}=10$, optical received power $=-5 \mathrm{dBm}$, bandwidth of $\mathrm{B}=5 \mathrm{GHz}$ has been plotted. Unlike Figure 6, here the BER is decreases with the increase of value of amplifier gain.

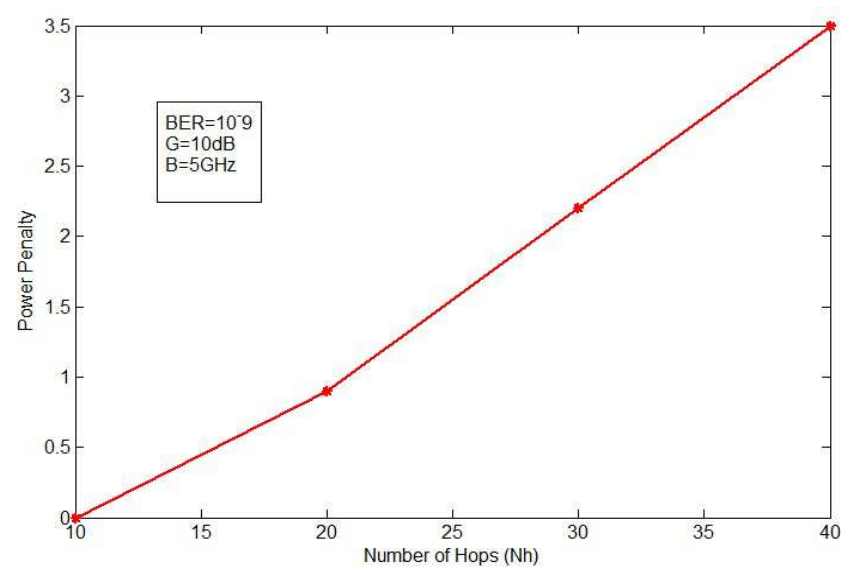

Figure 8. Power penalty versus number of hops for $B E R=10^{\wedge}-9$ with a fixed gain of $G=10 \mathrm{~dB}$ and bandwidth $B=5 \mathrm{GHz}$.

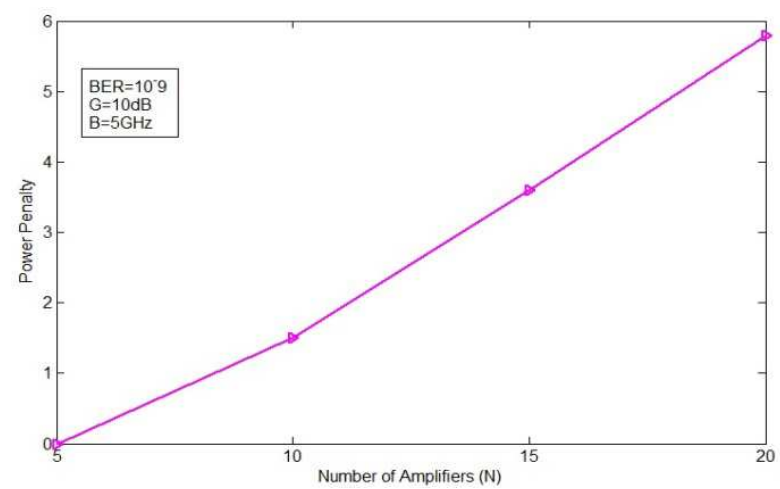

Figure 9. Power penalty vs No. of amplifiers for $B E R=10^{\wedge}-9$ with a fixed gain of $G=10 \mathrm{~dB}$ and bandwidth $B=5 \mathrm{GHz}$.
In Figure 8, the power penalty vs no. of hops has been demonstrated with a fixed gain. The power penalty increases with the increase of number of hops. In Figure 9, the power penalty has been plotted against number of amplifiers. So combining Figure 8 and Figure 9 it can be concluded that, power penalty increases with the increase of both the value of number of amplifiers and number of hops.

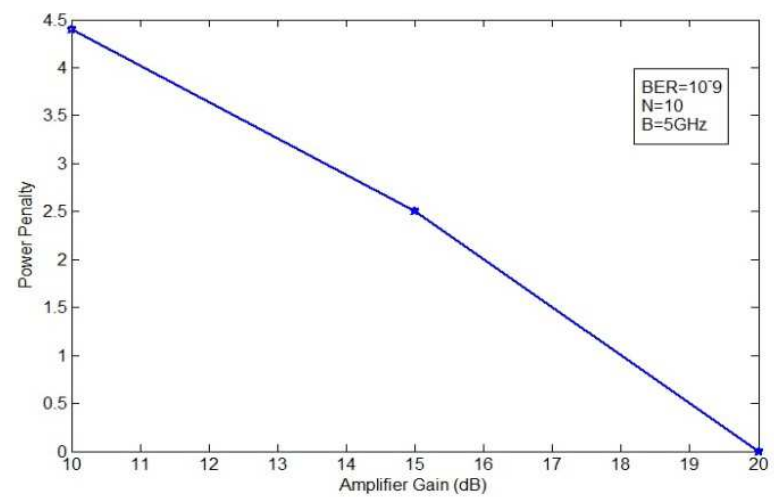

Figure 10. Power penalty vs amplifier gain for $B E R=10^{\wedge}-9$ for $N=10$, $B=5 G H z$.

In Figure 10, power penalty has been plotted against amplifier gain. It depicts that power penalty decreases with the increase of amplifier gain.

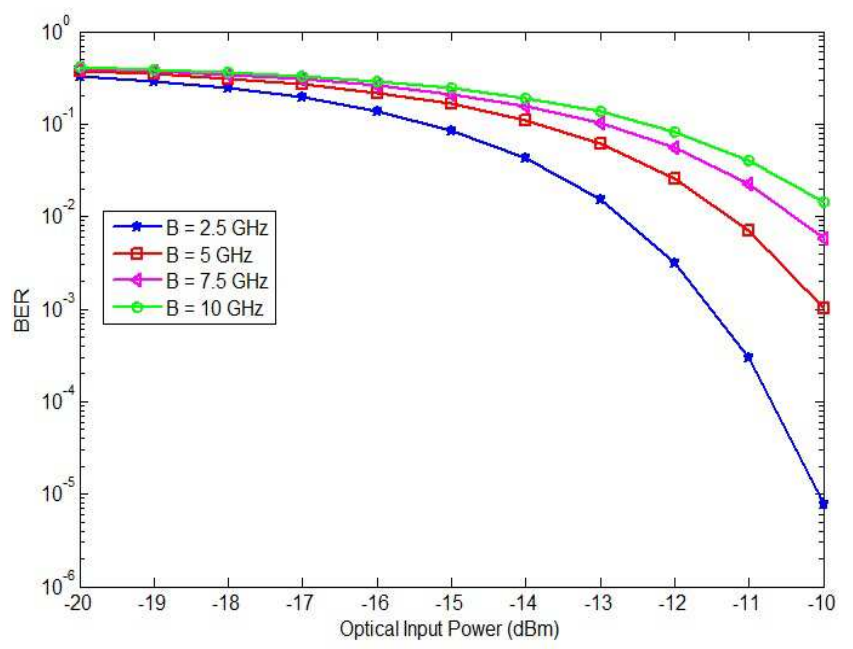

Figure 11. BER versus optical input power varying bandwidth for a fixed gain $10 \mathrm{~dB}$.

In Figure 11, BER has been demonstrated with an optical input power by varying different bandwidth. From the equation of (1, (2), (3) and (5) it can be seen that all the receiver noises and ASE noise are proportional to receiver bandwidth. So as the value of bandwidth is increases the SNR will be decreased and hence the BER will increase. In this figure BER is highest for $10 \mathrm{GHz}$ bandwidth. Therefore, a lower bandwidth produces a lower BER. It can be concluded that improved BER performance will be obtained when bandwidth is smaller. Also a small bandwidth corresponds to a smaller receiver size thus minimizes the cost. This figure is plotted for $\mathrm{P}-\mathrm{i}-\mathrm{N}$ receiver but this observation is valid for APD receiver also. 


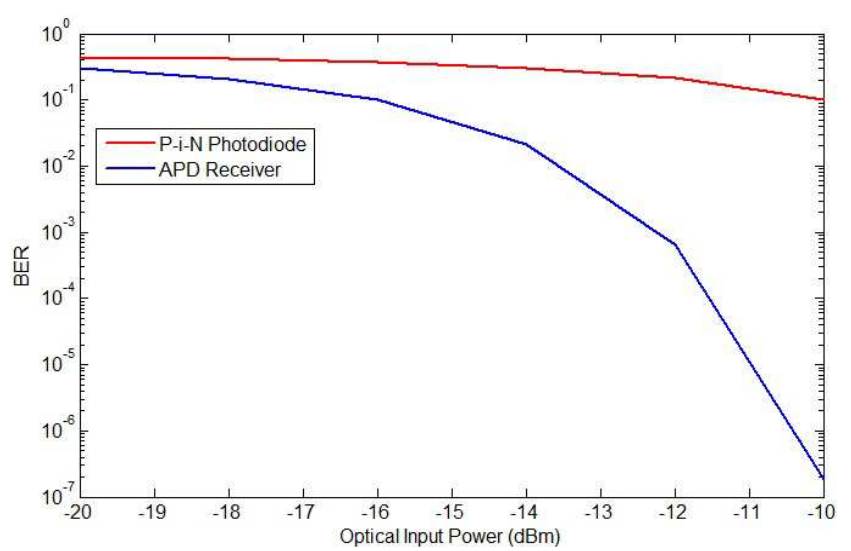

Figure 12. BER vs optical input power for $P-i-N$ and APD Receiver for a fixed bandwidth of $B=5 \mathrm{GHz}$.

In Figure 12, BER is plotted against optical received power for $\mathrm{P}-\mathrm{i}-\mathrm{N}$ receiver and APD receiver. From Equation (8) and (9) it is seen that when the value of APD gain is 1, the SNR expression will be identical for both optical receiver. From this figure it is observed that BER is lower for APD receiver and it can be concluded that APD provides better performance compared to $\mathrm{P}-\mathrm{i}-\mathrm{N}$ optical receiver.

The performance of APD receiver depends on APD gain (M). After a certain value of APD gain $(M=20 \mathrm{~dB})$, the improvement of DWDM system performance is not significant. We also identified the limitations of this article. From figure 11, for a given value of amplifier gain (10dB) the target BER $10^{-9}$ has not been achieved by varying receiver bandwidth. Therefore, in order to achieve desired threshold BER, the value of APD gain and receiver bandwidth should be optimum.

\section{Conclusion}

In this article, a general approach has been taken to evaluate the performance of DWDM system considering the effect of number of cascaded optical amplifiers in the presence of crosstalk. The SNR and BER have been analyzed by varying different parameters. Outcome of our investigation shows that, the system suffers from a power penalty which increases with the decrement in channel spacing and with the increment of optical amplifiers, and number of hops. The system performance can be substantially improved by using optimum number of optical amplifiers with higher gain and optimum receiver gain having moderate bandwidth. In future we expect to extend our analysis with intensity modulation coherent detection (IM/CD) and Raman amplifiers operating in a large wavelength as well as a wide bandwidth.

\section{Acknowledgements}

The author would like to express grateful thanks to his supervisor Dr. Satya Prasad Majumder, Professor, Dept. of EEE, BUET for his help, guidance and advice. The author also wishes to thank to Md. Mahfuzul Haque, Assistant Professor, BUBT, Bangladesh. The author's special thanks are sent to his father, mother and sister for their support and encouragement.

\section{References}

[1] Borella, M., Jue, J., Banerjee, D., Ramamurthy, B. and Mukherjee, B., "Optical components for WDM lightwave networks", Proceedings of the IEEE, Vol. 85 No. 8, August 1997, pp. 1274-1307.

[2] Bracket, C., "Dense wavelength division multiplexing networks: principles and applications", IEEE Journal Select. Areas Commun., August 1990, Vol. 8, No. 6, pp. 948-964.

[3] Jahid, A. and Alam, Z., "Performance analysis of DWDM system considering the effects of cascaded optical amplifiers with optimum receiver gain", American Journal of Engineering and Research, July 2015, Vol. 4, Issue 8, pp. 0108 .

[4] Tian, C. and Kinoshita, S., "Analysis and control of transient dynamics of EDFA pumped by 1480- and 980-nm lasers", IEEE/OSA Journal of Lightwave Technology, August 2003, vol. 21 , No. 8 , pp. 1728- 1734.

[5] Paraschis, L., Gerstel, O. and Frankel, M., "Metro networks: Services and technologies in optical fiber telecommunications, B: Systems and Networks", ed. Kaminow, I., Li, T. and Willner, A., Academic Press editors, EUA, 2008, ch. 12.

[6] E. lannone, and R. Sabella, "Optical path technologies: A comparison among different cross-connect architectures," Journal of Lightwave Technology, Oct. 1996, vol. 14, no. 10, pp. 2184-2194.

[7] Elrefaie, A., Goldstein, E., Zaidi, S. and Jackman, N., "Fiberamplifier cascades with gain equalization in multiwavelength unidirectional inter-office ring network," IEEE Photon. Technol. Lett., vol. 5, Sept. 1993, pp. 1026- 1031.

[8] Feuer, M., Kilper, D. and Woodward, S., "ROADMs and their system applications" in "Optical fiber telecommunications V, B: Systems and Networks", ed. Kaminow, I., T. and Willner, A., Academic press editors, EUA, 2008, ch.8.

[9] Dods S. D., Anderson T. B., "Calculation of bit-error rates and power penalties due to incoherent crosstalk in optical networks using Taylor series expansions," J. Lightwave Technol., vol. 23, April 2005, pp. 1828-1837.

[10] Maria Teresa Pinto, Ferreira Palma Ramalho, "Performance analysis of an optical link in DWDM sytems".

[11] Connely, M. J., "Semiconductor optical amplifiers", Kluwer Academic, 2002.

[12] Ramamurthiu, B., "Design of Optical WDM Networks, LAN, MAN, and WAN Architectures:, Kluvier, 2001.

[13] Ramaswami, R. and K. Sivarajan, "Optical Network: A Practical Perspective”,3rd edition, Morgan Kaufmann, 2002.

[14] Keiser, G., "Optical Fiber Comunications", $3^{\text {rd }}$ edition,McGraw-Hill, 2000.

[15] Kelvin B. A., Afa J. T., "Bit Error Rate Performance of Cascaded Optical Amplifiers Using Matlab Computation Software," European Scientific Journal, vol. 9, No. 3, January 2013. 
116 Abu Jahid et al: : Performance Analysis of DWDM System with Optical Amplifiers in Cascade Considering the Effect of Crosstalk

[16] H. Takahashi, K. Oda, and H. Toba, "Impact of crosstalk in an arrayed waveguide multiplexer on $\mathrm{NxN}$ optical interconnection", Journal of Lightwave Technology, vol. 14, no. 6, June 1996, pp. 1120-1126.

[17] S. D. Dods, J. P. R. Lacey, and R. S. Tucker, "Homodyne crosstalk in WDM ring and bus networks", IEEE Photonic Technology Letter, vol. 10, no. 3, Mar. 1998 pp. 457-458.

[18] K. P. Ho, C.K. Chan, F. Tong, and L.K. Chen, "Exact analysis of homodyne crosstalk induced penalty in WDM networks", IEEE Photonic Technology Letter, vol. 9, no. 3,Sept. 1997, pp. 1285-1287.
[19] Y. Shen, K. Lu, and W. Gu, "Coherent and incoherent crosstalk in WDM optical networks", Journal of Lightwave Technology, vol. 17, no. 5, May 1999, pp. 759-764.

[20] Senior, J.M. 'Optical fibre communication principles and practice" 3rd edition Prentice Hall 2009. 